\title{
Ethnomedicinal and phytochemical review of Pakistani medicinal plants used as antibacterial agents against Escherichia coli
}

\author{
Muhammad Adnan ${ }^{1 *}{ }^{*}$, Roqaia Bibi ${ }^{1 \dagger}$, Sakina Mussarat ${ }^{1+}$, Akash Tariq $^{1+}$ and Zabta Khan Shinwari ${ }^{2+}$
}

\begin{abstract}
Medicinal plants have always been part of human culture and have the potential to cure different diseases caused by microorganisms. In Pakistan, biologists are mainly focusing on plants' antimicrobial activities against Escherichia coli due to its increasing resistance to antibiotics. In total, extracts from 34 ethnomedicinally valuable Pakistani plants were reported for in-vitro anti-E. coli activities. Mostly methanolic extracts of medicinal plants were used in different studies, which have shown comparatively higher inhibitory activities against $E$. coli than n-hexane and aqueous extracts. It has been found that increasing concentration $(\mathrm{mg} / \mathrm{ml})$ of methanolic extract can significantly increase $(p<0.01)$ anti-E. coli activities. Not all medicinal plants are extracted in solvents others than above, which should also be tested against E. coli. Moreover, medicinal plant species must be fully explored phytochemically, which may lead to the development of new drugs.
\end{abstract}

Keywords: Medicinal plants, Biological screening, Ethnomedicines, Phytochemistry, Bacteria

\section{Introduction}

Therapeutic properties of medicinal plants are well recognized at global level [1]. As an estimate, over $50 \%$ of modern clinical drugs have natural products' origin [2]. World Health Organization has emphasized on the use of traditional medicines and reported about $80 \%$ of population from developing countries relies on medicinal plants for their primary health care [3,4]. It is believed that more than 8,000 plants species in South Asia carries medicinal properties, of which 1000 exists in Pakistan [5]. Local people use these medicinal plants for the treatment of various ailments through their indigenous knowledge [6]. However, due to modernization, traditional medicines are only practiced in remote rural areas $[7,8]$.

In Pakistan, pathogenic bacteria are causing serious infectious diseases like gastro-intestinal, pneumonia, pulmonary and skin related. A number of Pakistani medicinal plants have been tested for their antimicrobial activities [9]. These plants contain different phytochemicals such as

\footnotetext{
* Correspondence: ghurzang@hotmail.com

tEqual contributors

'Department of Botany, Kohat University of Science and Technology, Kohat 26000, Pakistan

Full list of author information is available at the end of the article
}

alkaloids, glycosides, saponins, resins, oleoresins, sesquiterpene lactones and oils (essential and fixed). Other compounds like furanocoumarins, hydroxycoumarins, napthoquinones, acylphloroglucinols and sterones have also been isolated from these species. It was identified that $74 \%$ of the 119 plant derived drugs were discovered as a result of isolation of active substances from medicinal plants [10].

Escherichia coli are gram negative bacteria, and mainly responsible for urinary tract and gastro-intestinal infections in human [11]. They are the best and most studied free-living microorganisms [12,13]. Some strains of $E$. coli live as harmless commensalism in animals' intestines while others causes serious diseases. These strains included enteropathogenic, enterohemorrhagic, enteroinvasive, enterotoxigenic, and enteroaggregative [14]. The enterohemorrhagic E. coli strain (EHEC) O157:H7 was first recognized as a gastro-intestinal pathogen in 1982 and became a world-wide public health problem [15]. However, most of the diseases caused by these bacteria are being treated locally using medicinal plants. Different methods like biological screening, isolation of compounds and clinical trials have been used to find out the efficacy 
of medicinal plants against microorganisms causing a particular disease $[16,17]$.

Emergence of multiple drug resistant bacterial strains due to indiscriminate use of antibiotics has generated a keen interest in the discovery of effective plants derived drugs [18]. E. coli are showing increased resistance to different antibiotics like amoxicillin and trimethoprim $[19,20]$. Hence, searching of alternative and effective medicines from plants against such resistant bacteria has become an important concern all over the world [21]. Antibiotics on one side became ineffective to bacterial strains but also costly for the poor communities of developing world $[22,23]$. Furthermore, the antibiotics may be associated with adverse effects including hypersensitivity and immune suppression [24]. Therefore, this review was designed with the aim to (i) compile the available fragmented literature on anti-E. coli effect of Pakistani medicinal plants, and (ii) suggest measures on newer and safer herbal drugs for the diseases caused by the E. coli. Furthermore, this review will provide knowledge on ethnomedicines and phytochemistry of those Pakistani medicinal plants having anti-E. coli potential. Above all, this review will provide baseline information for chemists, pharmacists and pharmacologists to carry out in-depth in-vitro and in-vivo activities for the development of novel drugs against $E$. coli with low cost and less side effects on living system.

\section{Methodology}

\section{Literature selection}

Online literature on antibacterial activities of Pakistani medicinal plants against $E$. coli was searched and gathered using online bibliographic databases including Google Scholar, ISI Web of Knowledge and Science Direct Navigator, as well as some libraries sources. An extensive number of published and unpublished articles and reports were found on Pakistani medicinal plants extracted with different solvents (methanol, ethanol, ethyl acetate, nhexane, chloroform etc.) for theirs in-vitro biological screening. In total, 112 plants were found tested for their in-vitro anti-E. coli in Pakistan. However, this review consisted of 34 plants, on which sufficient information were available regarding extracts' concentrations $(\mathrm{mg} / \mathrm{ml})$ necessary for maintaining uniformity in the data. This study is the combination of anti-E. coli activities, ethnomedicinal properties and phytochemistry of reported medicinal plants that were collected from the available literature.

\section{Extraction techniques used in Pakistan}

Extraction is the process of separation of active metabolites of medicinal plants using different solvents through standard procedures. Common techniques used in Pakistan for extraction process are Maceration, Infusion, Percolation, Decoction and Soxhlet $[25,26]$. Maceration is the most proffered technique, in which powdered plant-drug is kept in a container with solvent for a defined period with frequent stirring until soluble matter is dissolved [27].

\section{Data organization and statistical analysis}

Data was organized and tabulated using Microsoft Excel 2007 and Word 2007. First table was designed on the invitro anti-E. coli activities of Pakistani medicinal plants. This table consisted of data on the concentrations of plant extracts (uniformed to $\mathrm{mg} / \mathrm{ml}$ ) and their anti-E. coli zone of inhibition (uniformed to $\mathrm{mm}$ ). Second table composed of ethnomedicinal properties and phytochemistry of reported medicinal plants. Figure 1 depicts total number of medicinal plants used against $E$. coli in Pakistan, which justifies the criteria of species' selection for this review. Pearson correlation was applied using SPPS between plant extracts' concentrations and anti-E. coli zone of inhibition

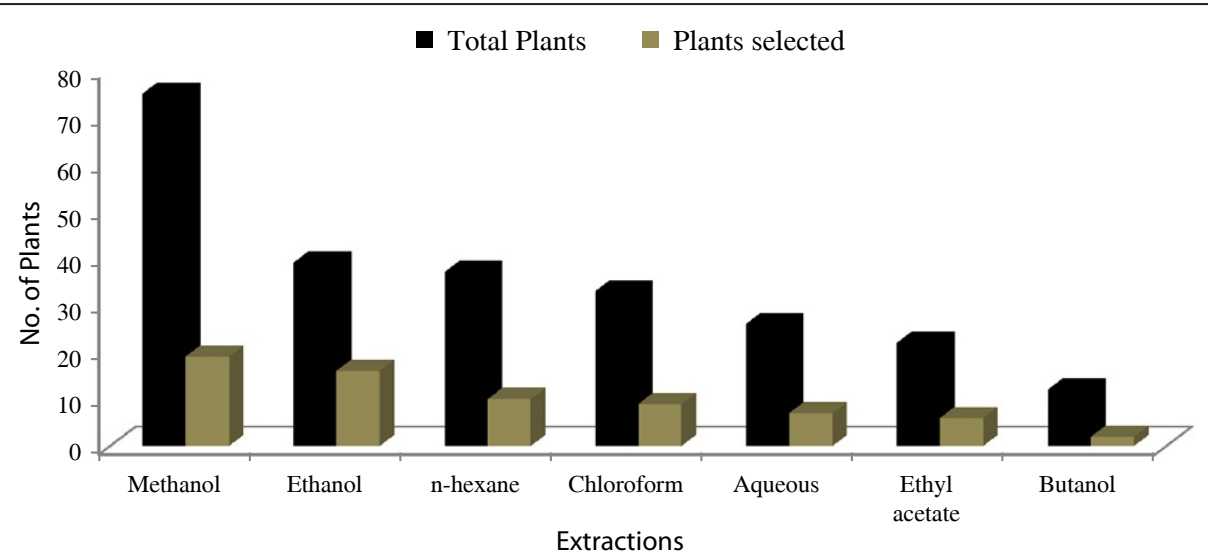

Figure 1 Pakistani medicinal plants extracted with different solvents. Plants selected indicate those that are selected out of total species for this review. 
(Figures 2 and 3). Furthermore, Figure 4 was developed in Chemdraw, which illustrates active phytochemical of selected medicinal plants having anti-E. coli activities.

\section{Discussion}

Medicinal plants extracted with methanol and ethanol

Methanol and ethanol are organic compounds used for the extractions of different medicinal plants. These solvents are mostly preferred throughout the world for extraction process [28]. Present review showed that majority of plant species (19) were extracted using methanol solvent (Figure 1). The higher use of methanol might be associated with its higher antibacterial activities in comparison to other solvents. Statistically plant extracts' of methanolic concentrations $(\mathrm{mg} / \mathrm{ml})$ in DMSO have significantly increased the anti-E. coli inhibitory activities (Figures 2 and 3).

Ethanolic extracts of certain plants also showed excellent inhibitory activities. Anti-E. coli inhibitory activities of methanol and ethanol might be related to their polar nature, due to which these solvents can easily degrade the cell wall of medicinal plants and helps in releasing polyphenols from cells. Ncube et al. [27] mentioned that polyphenols are best in their antibacterial activities. Polyphenols are organic in nature, which can be obtained through methanolic and ethanolic extractions [16].

\section{Withania somnifera}

$W$. somnifera is widely used as traditional medicine in remote areas of Pakistan for various ailments (Table 1). Mahmood et al. [29] described that methanolic extract of $W$. somnifera at different concentrations 15, 10, 5 and $3 \mathrm{mg} / \mathrm{ml}$ showed 8, 7, 3.7 and $1 \mathrm{~mm}$ inhibition against $E$. coli, respectively. Leaves extract of $W$. somnifera showed $18 \mathrm{~mm}$ inhibition against $E$. coli at $10 \mathrm{mg} / \mathrm{ml}$ concentration [30] (Table 2). These strong antibacterial activities of W. somnifera might be due to the presence of Withanolides (Figure 4.3), which have been isolated from the leaves [31] (Table 1).

\section{Justicia adhatoda}

$J$. adhatoda is traditionally being used for the treatment of variety of diseases caused by E. coli and other microorganisms (Table 1). Limited inhibition of methanolic extracts of its leaves was found against E. coli (Table 2). J. adhatoda showed $2 \mathrm{~mm}$ inhibition at $15 \mathrm{mg} / \mathrm{ml}$ concentration, $1.6 \mathrm{~mm}$ inhibition at $12.5 \mathrm{mg} / \mathrm{ml}$ concentration, $1.5 \mathrm{~mm}$ at $10 \mathrm{mg} / \mathrm{ml}$ and $1.4 \mathrm{~mm}$ at $7.5 \mathrm{mg} / \mathrm{ml}$
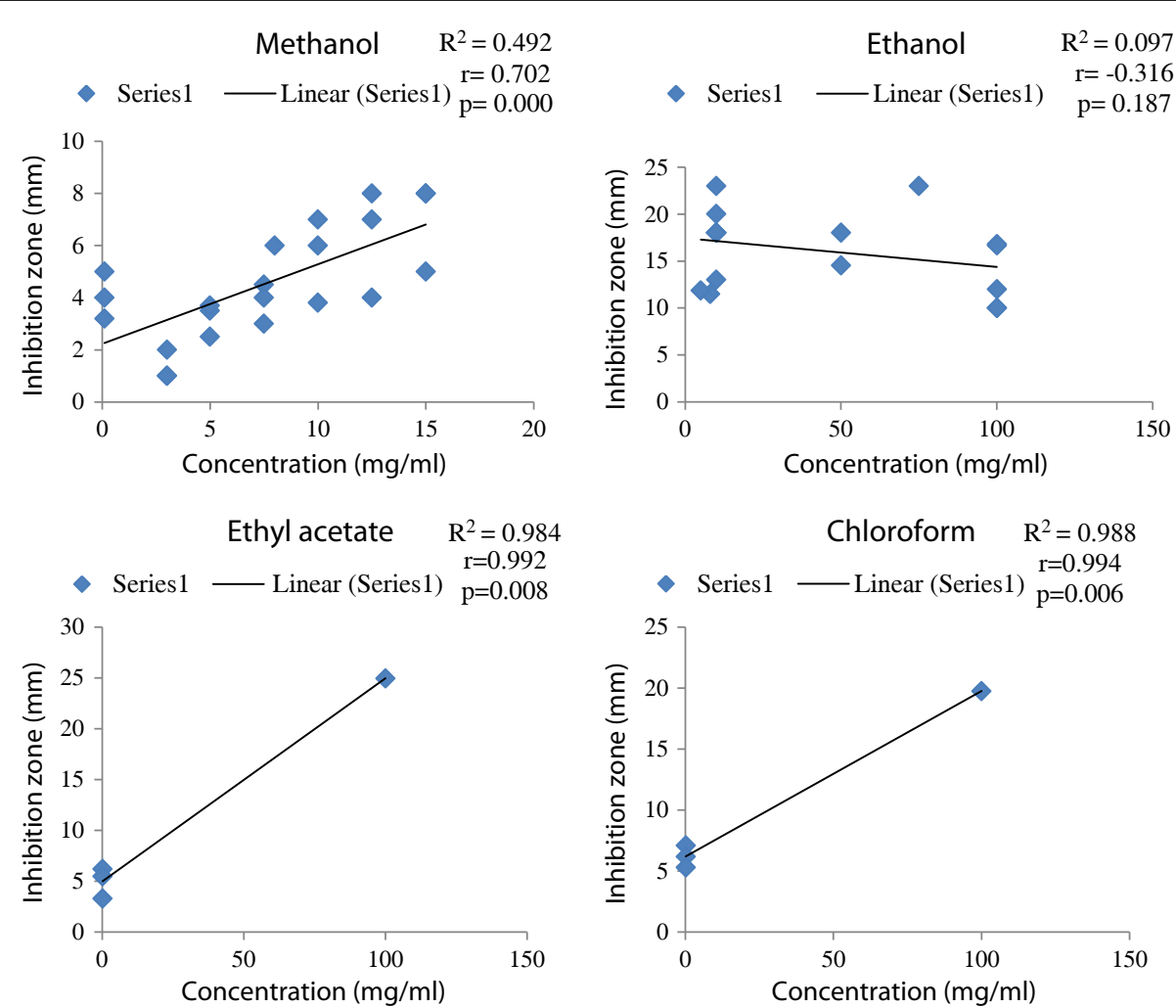

Figure 2 Pearson correlations between medicinal plant extracts' concentrations ( $\mathrm{mg} / \mathrm{ml})$ and inhibition zones of $E$. coli $(\mathrm{mm})$. 

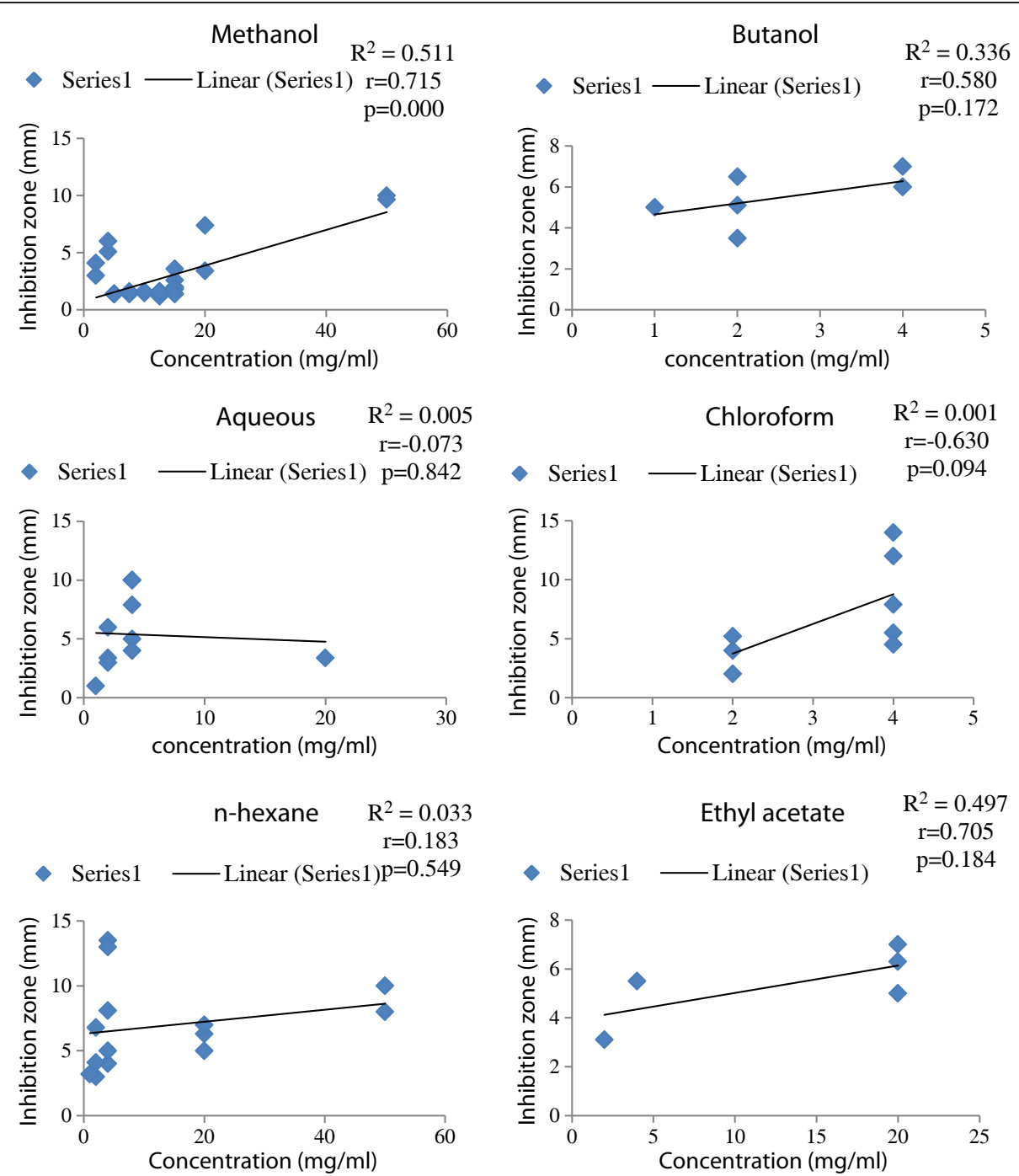

Figure 3 Pearson correlations of different concentration of medicinal plants extracts dissolved in DMSO solvent $(\mathrm{mg} / \mathrm{ml})$ and inhibition zones of $E$. coli $(\mathrm{mm})$.

concentration when dissolved in DMSO (Table 2). Studies have revealed the presence of alkaloids in the methanolic extract of its leaves (Table 1). Alkaloids isolated from J. adhatoda showed bronchodilator activity [86], however not evaluated for antimicrobial activities.

\section{Althaea officinalis}

Roots of $A$. officinalis are very useful and traditionally used for intestinal and respiratory problems. The methanolic extract of roots, leaves and flowers of $A$. officinalis when dissolved in DMSO have shown $1.9 \mathrm{~mm}$ inhibition against $E$. coli (Table 2). Phytoconstituents like altheahexacosanyl lactone (Figure 4.1), altheacalamene, $\beta$-sitositerol, altheacoumarin glucoside and other constituents have been obtained from the methanolic and ethanolic extract of root, seeds and leave of $A$. officinalis (Table 1). The anti-E. coli activity of this plant could be due to presence of these compounds. However, further studies are required in order to find out the constituents that may have strong potential against E. coli.

\section{Azadirachta indica}

Ethnomedicinally, A. indica is considered one of medicinal plants having great potential against variety of diseases (Table 1). For instance, the decoction of leaves is used for curing digestive and gastric problems. Leaves are dried, crushed and powder is mixed with small quantity of water and taken as remedies of freckles on face and increase appetite by lessening stomach flatulence and killing intestinal worms $[35,36]$. Ethanolic extract of leaves of $A$. indica showed 18 and $23 \mathrm{~mm}$ inhibition at 50 and $75 \mathrm{mg} / \mathrm{ml}$, respectively against $E$. coli [78]. More than 135 compounds have been isolated so far from different parts of $A$. indica, however not of them are 


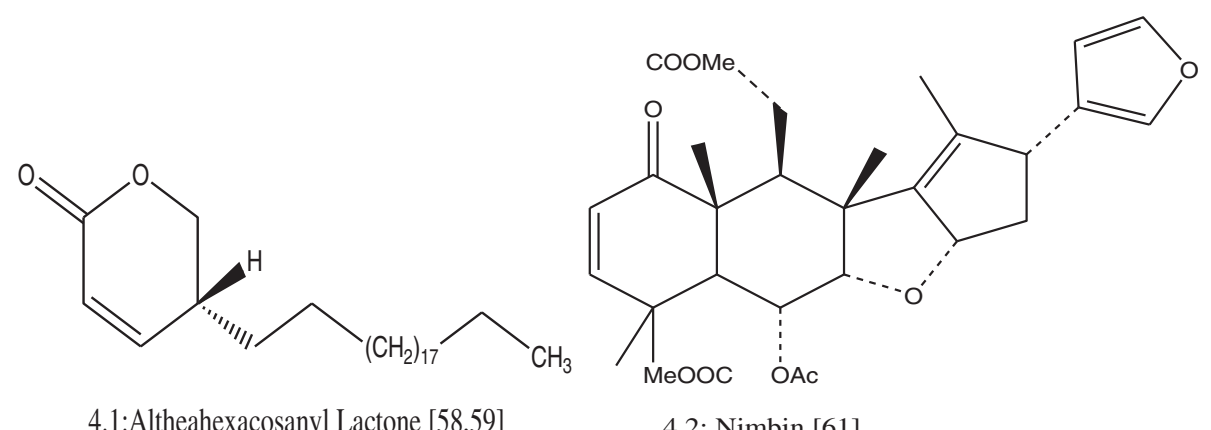

4.1:Altheahexacosanyl Lactone [58,59]

4.2: Nimbin [61]<smiles>[2H][C@@H]1c2ccccc2N=C2C(O)CCN21</smiles>

4.3: Alkaloids of J.adhatoda $[79,80]$

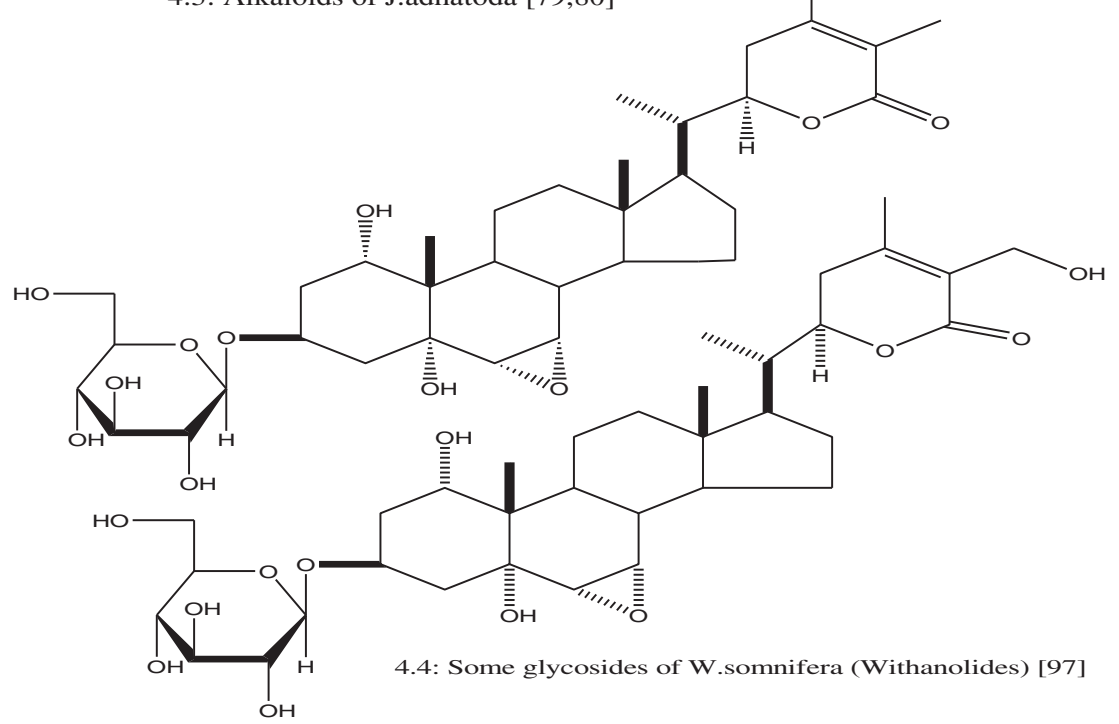

Figure 4 Structure of chemical compounds isolated from plants having antibacterial activities.

studied for their biological activities. Nimbin (Figure 4.2) and Nimbidin are major crude bitter principle extracted from the oil of seed kernels of $A$. indica, which have demonstrated several biological activities including antifungal, antibacterial and anti-inflammatory [76].

\section{Mentha longifolia}

Traditionally the decoction of $M$. longifolia is used for cholera, diarrhea and stomach problems in the rural area of Pakistan [61]. The ethanolic extract of leaves of $M$. longifolia showed $18 \mathrm{~mm}$ inhibition against $E$. coli when dissolved in their respective solvent at $10 \mathrm{mg} / \mathrm{ml}$ (Table 1).
Monoterpenes and sesquiterpenes present in aerial parts were found to possess antibacterial activities [87]. M. longifolia has also been scientifically proved for its insecticidal [87], antispasmodic and antiplatelet properties [88].

\section{Delonix regia}

Ethnomedicinally, the root of $D$. regia has been proved very potent against abdominal pain while leaves are used as anti-inflammation. In-vitro ethanolic extract of $D$. regia has shown $10 \mathrm{~mm}$ zone of inhibition at $100 \mathrm{mg} / \mathrm{ml}$ concentration against E. coli [12] (Table 2). Large number of phytoconstituents such as tannins, triterpenoids, 
Table 1 Ethnobotany and phytochemistry of Pakistani medicinal plants

\begin{tabular}{|c|c|c|c|c|c|c|c|}
\hline $\begin{array}{l}\text { Plant Species/ } \\
\text { Family names }\end{array}$ & Part used & Extract & Phytoconstituents & Ethnobotany & Mode of preparation & $\begin{array}{l}\text { Route of } \\
\text { admin. }\end{array}$ & References \\
\hline $\begin{array}{l}\text { Althaea officinalis } \\
\text { Linn Malvaceae }\end{array}$ & $\begin{array}{l}\text { Seed, root, } \\
\text { leaves, flower }\end{array}$ & Methanol & 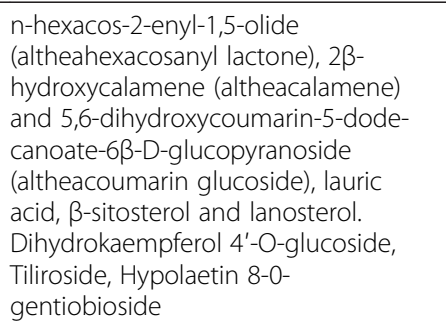 & $\begin{array}{l}\text { Expectorant, demulcent, } \\
\text { burns, snake bite, asthma, } \\
\text { bronchitis pneumonia, } \\
\text { rheumatism, kidney and } \\
\text { bladder problems }\end{array}$ & $\begin{array}{l}\text { Decoctions of the plant, especially of } \\
\text { the root, are very useful for intestinal } \\
\text { problems. Seeds, leaves and flowers } \\
\text { are boiled in wine or milk and taken } \\
\text { to relieve diseases like chest, coughs, } \\
\text { bronchitis and whooping-cough. }\end{array}$ & Oral, dermal & {$[32-34]$} \\
\hline $\begin{array}{l}\text { Azadirachta indica } \\
\text { Adr. Juss. } \\
\text { Meliaceae }\end{array}$ & Leaves & Ethanol & Azadirachtin & $\begin{array}{l}\text { Antiseptic, digestive and } \\
\text { gastric problems, skin } \\
\text { diseases, stomach flatulence }\end{array}$ & $\begin{array}{l}\text { Decoction of leaves is taken for } \\
\text { digestive and gastric problems. Fresh } \\
\text { leaves are boiled in water and tied } \\
\text { on wounds. Leaves are dried, } \\
\text { crushed and powder is mixed with } \\
\text { small quantity of water and taken for } \\
\text { the remedy of freckles on face and } \\
\text { increase appetite by lessening } \\
\text { stomach flatulence and killing } \\
\text { worms. }\end{array}$ & Oral, dermal & {$[35-37]$} \\
\hline \multirow[t]{2}{*}{$\begin{array}{l}\text { Calendula arvensis } \\
\text { L. Compositae }\end{array}$} & \multirow[t]{2}{*}{ Leaves } & \multirow[t]{2}{*}{ Ethanol } & $\begin{array}{l}\text { 28-O- } \beta \text {-D-glucopyranoside-3- } \beta \text {-O-(O- } \\
\beta \text {-D-galactopyranosyl }(1 \rightarrow 3)-\beta \text {-D- } \\
\text { glucopyranoside. }\end{array}$ & \multirow[t]{2}{*}{$\begin{array}{l}\text { Hepatitis and spleen } \\
\text { enlargement control }\end{array}$} & \multirow[t]{2}{*}{$\begin{array}{l}\text { Decoction of leaves is used as } \\
\text { required. }\end{array}$} & \multirow[t]{2}{*}{ Oral } & \multirow[t]{2}{*}[35,38]{} \\
\hline & & & $\begin{array}{l}\text { 3- } \beta \text {-O-(O- } \beta \text {-D-galactopyranosyl }(1 \rightarrow \\
\text { 3)- } \beta \text {-D-glucopyranoside. }\end{array}$ & & & & \\
\hline $\begin{array}{l}\text { Calotropis procera } \\
\text { Ait. f., Hort. } \\
\text { Solanaceae }\end{array}$ & Stem, leaves & $\begin{array}{l}\text { Methanol, } \\
\text { aqueous }\end{array}$ & $\begin{array}{l}\text { alkaloids, flavonoids, tannins, steroids, } \\
\text { triterpenoids, saponins }\end{array}$ & $\begin{array}{l}\text { Expectorant, anthelmentic, } \\
\text { cholera, asthma, earache, } \\
\text { pyorrhea, gastro-intestinal } \\
\text { diseases }\end{array}$ & $\begin{array}{l}\text { Stem latex is used in earache and } \\
\text { asthma. Infusion of leaves used for } \\
\text { stomach problems. }\end{array}$ & Oral, dermal & {$[35,39]$} \\
\hline \multirow{4}{*}{$\begin{array}{l}\text { Cannabis sativa } \mathrm{L} \text {. } \\
\text { Cannabaceae }\end{array}$} & \multirow[t]{4}{*}{ Leaves } & \multirow[t]{4}{*}{ Ethanol } & Anhydrocannabisativine & \multirow[t]{4}{*}{ Sedative, anodyne, narcotic } & \multirow{4}{*}{$\begin{array}{l}\text { Whole plant extract is effective cure } \\
\text { of livestock dysentery. Cannabis is } \\
\text { also used for the treatment of } \\
\text { number of condition including AIDS, } \\
\text { multiple sclerosis and thermotherapy } \\
\text { induced nausea. Its decoction is } \\
\text { used for the treatment of the cancer, } \\
\text { neuro protection, fever and high } \\
\text { blood pressure. It cause hallucination } \\
\text { when drunk in excessive quantity. }\end{array}$} & \multirow[t]{4}{*}{ Oral, dermal } & \multirow[t]{4}{*}[40-42]{} \\
\hline & & & Cannabisativine & & & & \\
\hline & & & cannabinoids & & & & \\
\hline & & & $\begin{array}{l}N \text {-(p-hydroxy- } \beta \text {-phenylethyl)-p- } \\
\text { hdroxy-(trans)-cinnamide }\end{array}$ & & & & \\
\hline $\begin{array}{l}\text { Carum copticum L. } \\
\text { Apiaceae }\end{array}$ & & $\begin{array}{l}\text { Methanol, ethanol, } \\
\text { n-hexane, acetone }\end{array}$ & & $\begin{array}{l}\text { Appetizers, kidney stone, } \\
\text { digestion and whooping } \\
\text { cough }\end{array}$ & $\begin{array}{l}\text { Seeds are taken with little salt for } \\
\text { gas trouble as stomach tonic. }\end{array}$ & Oral & {$[36,43]$} \\
\hline
\end{tabular}


Table 1 Ethnobotany and phytochemistry of Pakistani medicinal plants (Continued)

\begin{tabular}{|c|c|c|c|c|c|c|c|}
\hline \multirow[t]{3}{*}{$\begin{array}{l}\text { Cichorium Intybus } \\
\text { L. Asteraceae }\end{array}$} & \multirow[t]{3}{*}{ Root } & \multirow[t]{3}{*}{ Methanol } & 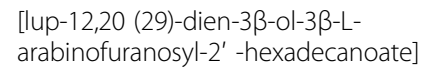 & \multirow[t]{3}{*}{ Abdominal pain, diarrhea } & \multirow[t]{3}{*}{$\begin{array}{l}\text { The whole plant is used for } \\
\text { carminative purposes. }\end{array}$} & \multirow[t]{3}{*}{ Oral } & \multirow[t]{3}{*}[34,40,44]{} \\
\hline & & & $\begin{array}{l}\text { [lup-12,20 (29)-dien-3ß-olyl } \\
\text { hexadecanoate] }\end{array}$ & & & & \\
\hline & & & $\begin{array}{l}\text { [4 } \beta \text {-(pent-2-enylolactone)- } \\
\text { hexatriacontane] }\end{array}$ & & & & \\
\hline $\begin{array}{l}\text { Cinnamomum } \\
\text { zeylanicum Blume. } \\
\text { Lauracaeae }\end{array}$ & Kohat & n-hexane & $\begin{array}{l}\text { carbohydrates, alkaloids, tannins, } \\
\text { steroids, tannins, flavonoids, } \\
\text { glycosides }\end{array}$ & $\begin{array}{l}\text { Toothache and sore gums, } \\
\text { carminative, stimulant, anti- } \\
\text { microbial, anti-fungal }\end{array}$ & $\begin{array}{l}\text { Bark is boiled in water and makes } \\
\text { tea and sipped. }\end{array}$ & Oral & {$[45]$} \\
\hline $\begin{array}{l}\text { Cistanche tubulosa } \\
\text { (Schenk) R. Wight. } \\
\text { Orobanchaceae }\end{array}$ & Stem & Methanol & Glycosides, monoterpenes & & & & {$[46]$} \\
\hline $\begin{array}{l}\text { Datura innoxia } \\
\text { Mill. Solanaceae }\end{array}$ & Seed & Methanol & 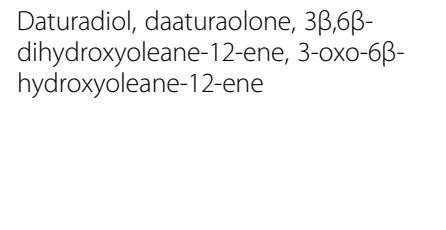 & $\begin{array}{l}\text { anti-inflammatory, laxative } \\
\text { antispasmodic, sedative, } \\
\text { malaria }\end{array}$ & $\begin{array}{l}\text { Smoke of the plant is inhaled to cure } \\
\text { asthma. Fruits are used in malaria. } \\
\text { Fruit is used to reveal cardiac pains } \\
\text { and distress. Leaves are used in } \\
\text { earache. The juice of the fruit is } \\
\text { applied to the scalp for curing } \\
\text { dandruff and falling hairs. }\end{array}$ & Dermal & {$[34,35,47]$} \\
\hline $\begin{array}{l}\text { Delonix regia } \mathrm{L} \text {. } \\
\text { Leguminosae }\end{array}$ & $\begin{array}{l}\text { Stem, bark, } \\
\text { leaves }\end{array}$ & Ethanol, methanol & $\begin{array}{l}\text { L-Azetidine-2-carboxylic acid, lupeol, } \\
\text { epilupeol, b-sitosterol, stigmasterol } \\
\text { and p- methoxybenzaldehyde alka- } \\
\text { loids, tannnins, triterpenoids, steroids, } \\
\text { glycosides, flavonoids, so-flaflavones, } \\
\text { flavones, anthocyanine, coumarines, } \\
\text { lignins, vitamin-A, vitamin-E, vitamin- } \\
\text { C.B-Amyrin, hesperitin }\end{array}$ & $\begin{array}{l}\text { Abdominal pains, bronchitis } \\
\text { and pneumonia }\end{array}$ & $\begin{array}{l}\text { Root decoction is used for } \\
\text { abdominal pains and in the } \\
\text { treatment of scorpion bite. The } \\
\text { leaves extract is used as anti- } \\
\text { inflammatory. The herb is also used } \\
\text { in bronchitis and pneumonia in in- } \\
\text { fants. It is used as a carminative. }\end{array}$ & Oral & {$[48-50]$} \\
\hline $\begin{array}{l}\text { Dodonaea viscosa } \\
\text { L. Capparidaceae }\end{array}$ & $\begin{array}{l}\text { Leaves, aerial } \\
\text { parts }\end{array}$ & Ethanol & $\begin{array}{l}\text { Tannins, saponins, flavanoids and } \\
\text { terpenoids }\end{array}$ & $\begin{array}{l}\text { Astringent, anti rheumatic, } \\
\text { swelling cutaneous, skeletal } \\
\text { and gastro-intestinal diseases } \\
\text { and burns }\end{array}$ & $\begin{array}{l}\text { Grind the leaves and add small } \\
\text { amount of water (Infusion) to make } \\
\text { fine paste for dermal use. }\end{array}$ & Oral, dermal & {$[40,51]$} \\
\hline $\begin{array}{l}\text { Eucalyptus } \\
\text { camaldulensis } \\
\text { Dehnh. Myrtaceae }\end{array}$ & Leaves & Ethanol & $\begin{array}{l}\text { Ellagitannins, flavonoids, } \\
\text { phloroglucinol derivatives and } \\
\text { galloyl esters. }\end{array}$ & Flu and cold & $\begin{array}{l}\text { Five to ten leaves boiled in water } \\
\text { and decoction is taken for flu twice a } \\
\text { day. }\end{array}$ & Oral & {$[36,52]$} \\
\hline $\begin{array}{l}\text { Ficus carica } \mathrm{L} \text {. } \\
\text { Moraceae }\end{array}$ & Leaves, fruit & Ethanol & $\begin{array}{l}\text { Steroids, triterpenoids, cumarines, } \\
\text { flavanoids and glycoside }\end{array}$ & $\begin{array}{l}\text { Respiratory, gastro intestinal, } \\
\text { urinary and cutaneous } \\
\text { diseases, demulcent, laxative, } \\
\text { antiseptic, constipation, } \\
\text { flatulence, measles, dysentery, } \\
\text { bladder problems and } \\
\text { verrucas }\end{array}$ & $\begin{array}{l}\text { Burn the leaves and the ash is } \\
\text { sprayed on the wounds dermally. } \\
\text { Decoction is used for intestinal } \\
\text { problems. Fresh fruit used in anemia } \\
\text { and constipation. Latex obtained } \\
\text { from the stem and leaves for } \\
\text { checking bleeding. }\end{array}$ & Oral, dermal & {$[34,53]$} \\
\hline $\begin{array}{l}\text { Glycyrrhiza glabra } \\
\text { L. Leguminosae }\end{array}$ & Root & Methanol & Glycyrrhizin & Respiratory illness, cough & $\begin{array}{l}\text { Dried root are crushed and powder } \\
\text { taken orally. }\end{array}$ & Oral & {$[54]$} \\
\hline
\end{tabular}


Table 1 Ethnobotany and phytochemistry of Pakistani medicinal plants (Continued)

\begin{tabular}{|c|c|c|c|c|c|c|c|}
\hline $\begin{array}{l}\text { Hyssopus officinalis } \\
\text { L. Lamiaceae }\end{array}$ & Leaves & Methanol & $\begin{array}{l}\text { a-Glucosidase inhibitors. quercetin 7- } \\
\text { O- } \beta \text {-D-apiofuranosyl- }(1 \rightarrow 2)-\beta-D-x y- \\
\text { lopyranoside and quercetin 7-O- } \beta \text {-D- } \\
\text { apiofuranosyl-( }(1 \rightarrow 2)-\beta-D \text {-xylopyran- } \\
\text { oside3'-O- } \beta \text {-D-glucopyranoside }\end{array}$ & $\begin{array}{l}\text { asthma, cough, bronchitis, } \\
\text { fever, trauma, rheumatism }\end{array}$ & & & {$[55,56]$} \\
\hline $\begin{array}{l}\text { Justicia adhatoda } \\
\text { L. Acanthaceae }\end{array}$ & Leaves & Methanol & Alkaloids & $\begin{array}{l}\text { Diuretic, jaundice, } \\
\text { antispasmodic cough, } \\
\text { asthma, bronchitis, } \\
\text { tuberculosis, rheumatism, } \\
\text { gastro-intestinal, diarrhea, } \\
\text { dysentery, antimicrobial }\end{array}$ & $\begin{array}{l}\text { Grind the leaves and mix it with } \\
\text { honey. The paste is used dermally } \\
\text { around the swelling. Decoction is } \\
\text { used for respiratory diseases and } \\
\text { diarrhea. }\end{array}$ & Oral, dermal & [57-59] \\
\hline $\begin{array}{l}\text { Malva neglecta } \\
\text { Wall. Malvaceae }\end{array}$ & & Methanol & & Purgative & & & {$[40]$} \\
\hline $\begin{array}{l}\text { Malva sylvestris } L . \\
\text { Malvaceae }\end{array}$ & $\begin{array}{l}\text { Leaves, root, } \\
\text { flower }\end{array}$ & Methanol & $\begin{array}{l}\text { (2-methyl-3-methoxy-5,6-dihydroxy- } \\
\text { 1,4-naphthoquinone) }\end{array}$ & & & & {$[60]$} \\
\hline $\begin{array}{l}\text { Mentha longifolia } \\
\text { L. Lamiaceae }\end{array}$ & Leaves & Ethanol & $\begin{array}{l}\text { Longifone, (longiside-A and -B) and } \\
\text { flavanone-glycoside (longitin) tricetin } \\
\text { 7-O-methylether 3'-O-glucoside 5'-O- } \\
\text { rhamnoside, tricetin 3'-O-glucoside } \\
\text { 5'-O-rhamnoside and tricetin 3'-O- } \\
\text { rhamnosyl- } 1 \rightarrow 4 \text {-rhamnoside }\end{array}$ & $\begin{array}{l}\text { Carminative, diarrhea, } \\
\text { dysentery and stomachache }\end{array}$ & $\begin{array}{l}\text { The dried plant is use for the } \\
\text { treatment of diarrhea and its } \\
\text { "chatenii" is good tonic for } \\
\text { improvement of stomach. The tea of } \\
\text { leaf with lemon extract is common } \\
\text { household tonic to cure cold, flu, } \\
\text { respiratory disorders. }\end{array}$ & Oral & {$[40,61,62]$} \\
\hline $\begin{array}{l}\text { Olea europaea. L } \\
\text { Oleaceae }\end{array}$ & Leaves & Ethanol & $\begin{array}{l}\text { Oleuropein, Hydroxytyrosol, } \\
\text { Leteoline-7-glucoside }\end{array}$ & & & & {$[63,64]$} \\
\hline $\begin{array}{l}\text { Otostegia limbata } \\
\text { (Benth.) Boiss } \\
\text { Labiatae }\end{array}$ & Leaves, root & Ethanol, methanol & 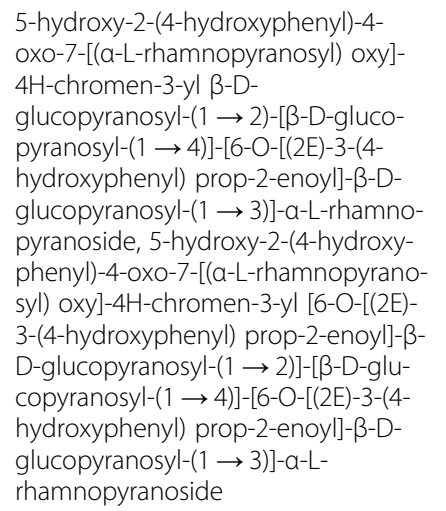 & $\begin{array}{l}\text { Wounds, gum diseases, } \\
\text { dental, cutaneous diseases }\end{array}$ & & Oral & {$[35,65]$} \\
\hline $\begin{array}{l}\text { Paeonia emodi } \\
\text { Wall. ex Royle } \\
\text { Paeoniaceae }\end{array}$ & $\begin{array}{l}\text { Root, flower, } \\
\text { leaves }\end{array}$ & Methanol & $\begin{array}{l}\text { Monoterpenes, monoterpene } \\
\text { glycosides, triterpenoids, flavonoids, } \\
\text { phenols and tannins }\end{array}$ & $\begin{array}{l}\text { Backache, epilipsy, } \\
\text { convulsions, uterine diseases, } \\
\text { vomiting, cholera, whooping } \\
\text { cough, diarrhea }\end{array}$ & $\begin{array}{l}\text { The leaves of Ruta graveolens, } \\
\text { Paeonia emodi root, are grounded } \\
\text { together and sieved through a cloth. } \\
\text { Mamordica charantia and water are } \\
\text { mixed together and added to sufuf } \\
\text { formed. }\end{array}$ & Oral, dermal & {$[34,66-68]$} \\
\hline
\end{tabular}

Otostegia limbata

(Benth.) Boiss

Labiatae

Monoterpenes, monoterpene glycosides, triterpenoids, flavonoids, vomiting, cholera, whooping formed. 4H-chromen-3-yl $\beta$-D-

Backache, epilipsy, cough, diarrhe The paste is used dermally around the swelling. Decoction is used for respiratory diseases and tuberculosis, rheumatism, dysentery, antimicrobial

Purgative 6-68] 
Table 1 Ethnobotany and phytochemistry of Pakistani medicinal plants (Continued)

\begin{tabular}{|c|c|c|c|c|c|c|c|}
\hline $\begin{array}{l}\text { Phyllanths emblica } \\
\text { L. Euphorbiaceae }\end{array}$ & Fruit, leaves & Methanol & $\begin{array}{l}\text { kaempferol-3-O-a-L-(6"-methyl)- } \\
\text { rhamnopyranoside, kaempferol-3-O- } \\
\text { a-L-(6"--thyl)-rhamnopyranoside 5- } \\
\text { hydroxymethylfurfural Qeurcetin, } \\
\text { gallicacid }\end{array}$ & $\begin{array}{l}\text { Carminative, stomachic, } \\
\text { diuretic, laxative cooling } \\
\text { effect, asthma, bronchitis, } \\
\text { scurvy, cardiac, tuberculosis, } \\
\text { diabetes, gonorrhea, } \\
\text { rheumatism, jaundice, } \\
\text { dysentery, diarrhea }\end{array}$ & $\begin{array}{l}\text { Dried fruits are grind and taken with } \\
\text { water against dysentery and diarrhea }\end{array}$ & Oral & {$[34,69,70]$} \\
\hline $\begin{array}{l}\text { Ricinus communis } \\
\text { L. Euphorbiaceae }\end{array}$ & Leaves, seed & Ethanol & $\begin{array}{l}\text { DPPH (I,I-diphenyl-2-picrylhydrazyl), } \\
\text { Gallic acid, quercetin, gentisic acid, } \\
\text { rutin, epicatechin and ellagic acid }\end{array}$ & $\begin{array}{l}\text { Emetic, narcotic, purgative, } \\
\text { swelling, prolapse of uterus, } \\
\text { gastro-intestinal diseases, } \\
\text { rheumatism, paralysis, } \\
\text { asthma, cough and } \\
\text { constipation }\end{array}$ & $\begin{array}{l}\text { Seed oil mixed with decoction of } \\
\text { jaman (Cordia Oblique) leaves is } \\
\text { given to cattle for constipation } \\
\text { problems and increase appetite. Its } \\
\text { leaf extract with (Grewia sp) bark } \\
\text { fiber and fruit is frequently used for } \\
\text { prolapse of uterus and easy delivery } \\
\text { and to hasten release of birth in } \\
\text { cattle. }\end{array}$ & Oral, dermal & {$[34,40,71]$} \\
\hline $\begin{array}{l}\text { Solanum } \\
\text { surrattense Burrn. f. } \\
\text { Solanaceae }\end{array}$ & Whole plant & Methanol & & $\begin{array}{l}\text { Chest pain, vomiting, burning } \\
\text { feet, cough, asthma, } \\
\text { expectorant, stomachache, } \\
\text { diuretic, gonorrhea, urinary, } \\
\text { gastro-intestinal diseases }\end{array}$ & $\begin{array}{l}\text { Fruit is dried, crushed and powder is } \\
\text { taken for abdomen pain and gas } \\
\text { trouble. }\end{array}$ & Oral & {$[29,36,59]$} \\
\hline $\begin{array}{l}\text { Solanum } \\
\text { xanthocarpum } \\
\text { Schrad. and } \\
\text { Wendl. Sert. } \\
\text { Hanov. Solanaceae }\end{array}$ & $\begin{array}{l}\text { Leaves, stem, } \\
\text { flower, root }\end{array}$ & Ethanol & $\begin{array}{l}\text { Carpesterol and four steroidal } \\
\text { glycosides, alkaloids, sterols, } \\
\text { saponine, flavonoids, glycosides }\end{array}$ & & & & {$[72,73]$} \\
\hline $\begin{array}{l}\text { Trigonella foenum } \\
\text { graecum } L \text {. } \\
\text { Leguminosae }\end{array}$ & $\begin{array}{l}\text { Seeds, stem, } \\
\text { Leaves }\end{array}$ & Ethanol & $\begin{array}{l}5,7,3^{\prime} \text {-trihydroxy-5'- } \\
\text { methoxylisoflavone, biochanin A, } \\
\text { formononetin, irilone, tricin, daidzein, } \\
\text { calycosin, orientin-2"-O-p-trans-cou- } \\
\text { marate, vitexin-2"-O-p-trans-couma- } \\
\text { rate, and tricin-7-O-B-D- } \\
\text { glucopyranoside }\end{array}$ & $\begin{array}{l}\text { anticancer, anti-inflammatory, } \\
\text { antiseptic, aphrodisiac, astrin- } \\
\text { gent, anthelmintic, wound } \\
\text { healing, gastroprotective, } \\
\text { chronic cough, leprosy, heart } \\
\text { disease, antidiabetic, diarrhea, } \\
\text { urethera prolapse }\end{array}$ & $\begin{array}{l}200 \mathrm{~g} \text { seeds are ground and the } \\
\text { resulting powder is used orally after } \\
\text { washing urethra with a sugar and } \\
\text { potash alum (potassium alum) } \\
\text { mixture for } 4-5 \text { days. } 50 \mathrm{~g} \text { seeds are } \\
\text { mixed with fodder and fed to animal } \\
\text { for 3-4 days. }\end{array}$ & Oral & {$[34,74,75]$} \\
\hline $\begin{array}{l}\text { Viscum album } \mathrm{L} . \\
\text { Loranthaceace }\end{array}$ & Leaves, twigs & $\begin{array}{l}\text { Ethyl acetate, } \\
\text { chloroform, } \\
\text { ethanolic, } \\
\text { methanolic, } \\
\text { aqueous }\end{array}$ & 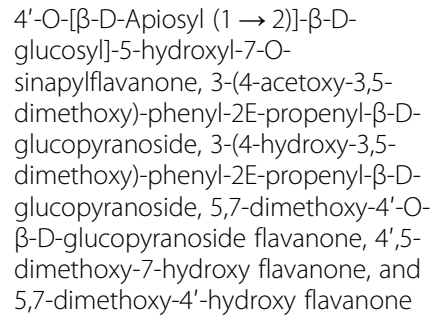 & $\begin{array}{l}\text { Anti-inflammatory, emetic, } \\
\text { purgative, anti - diabetic, } \\
\text { hernia }\end{array}$ & & & {$[76]$} \\
\hline $\begin{array}{l}\text { Withania } \\
\text { somnifera Dunal. } \\
\text { Solanaceae }\end{array}$ & Fruit, leaves & $\begin{array}{l}\text { Methanol, } \\
\text { ethanolic }\end{array}$ & withanosides I, II, III, IV, V, VI, and VII & $\begin{array}{l}\text { Anthelmintic, leucorrhoea, } \\
\text { tuberculosis abdominal pain }\end{array}$ & $\begin{array}{l}\text { Fruit is given to children for } \\
\text { removing abdominal pain. Decoction } \\
\text { is used for blood purification. }\end{array}$ & Oral & {$[35,31]$} \\
\hline
\end{tabular}


Table 1 Ethnobotany and phytochemistry of Pakistani medicinal plants (Continued)

\begin{tabular}{|c|c|c|c|c|c|c|c|}
\hline $\begin{array}{l}\text { Ziziphus vulgaris } \\
\text { Miller. } \\
\text { Rhamnaceae }\end{array}$ & Fruit & Methanol & $\begin{array}{l}\text { 3-O-robinobioside, quercetin 3-O- } \\
\text { rutinoside, 3-O-a-L-arabinosyl-(1 } \rightarrow \\
\text { 2)-a-L-rhamnoside, 3-O- } \beta \text {-D-xylosyl- } \\
(1 \rightarrow 2)-a-L-\text {-rhamnoside, 3', } 5^{\prime} \text {-di-C- } \beta- \\
\text { D-glucosylphloretin, 3-O- } \beta \text {-D-xylosyl- } \\
(1 \rightarrow 2) \text { - }- \text {-L-rhamnoside-4'-O- } a-L- \\
\text { rhamnoside, }\end{array}$ & $\begin{array}{l}\text { Laxative, cutaneous and } \\
\text { gastro-intestinal diseases }\end{array}$ & Infusion & Oral & {$[29,35]$} \\
\hline
\end{tabular}


Table 2 Antibacterial activities of Pakistani medicinal plants against $E$. coli at different concentration

\begin{tabular}{|c|c|c|c|c|c|c|}
\hline Plant Species & Location & Part used & Extract & $\begin{array}{l}\text { Concentration } \\
(\mathrm{mg} / \mathrm{ml})\end{array}$ & $\begin{array}{l}\text { Zone of } \\
\text { inhibition }(\mathrm{mm})\end{array}$ & References \\
\hline A. officinalis & Muzaffarabad & $\begin{array}{l}\text { Root, leaves, } \\
\text { flower }\end{array}$ & Methanol & $15(\mathrm{D})$ & 1.9 & [77] \\
\hline \multirow[t]{2}{*}{ A. indica } & \multirow[t]{2}{*}{ Faisalabad } & \multirow[t]{2}{*}{ Leaves } & \multirow[t]{2}{*}{ Ethanolic } & $50(C)$ & 18 & \multirow[t]{2}{*}{ [78] } \\
\hline & & & & $75(C)$ & 23 & \\
\hline C. arvensis & $\begin{array}{l}\text { Cherat, Mardan, } \\
\text { Malakand, Kohat }\end{array}$ & Leaves & Ethanolic & $10(C)$ & 18 & [30] \\
\hline \multirow[t]{18}{*}{ C. procera } & \multirow[t]{18}{*}{ Kohat } & \multirow[t]{10}{*}{ Stem } & n-hexane & $4(D)$ & 4 & \multirow[t]{18}{*}{ [17] } \\
\hline & & & Methanol & 4 (D) & 5.1 & \\
\hline & & & Aqueous & 4 (D) & 5 & \\
\hline & & & Chloroform & 4 (D) & 5.5 & \\
\hline & & & Butanol & 4 (D) & 6 & \\
\hline & & & n-hexane & 2 (D) & 3 & \\
\hline & & & Methanol & $2(D)$ & 3 & \\
\hline & & & Aqueous & 2 (D) & 3.4 & \\
\hline & & & Chloroform & $2(D)$ & 2 & \\
\hline & & & Butanol & $2(D)$ & 3.5 & \\
\hline & & \multirow[t]{8}{*}{ Leaves } & n-hexane & $4(D)$ & 8.1 & \\
\hline & & & Aqueous & 4 (D) & 7.9 & \\
\hline & & & chloroform & 4 (D) & 7.9 & \\
\hline & & & Butanol & $4(D)$ & 6 & \\
\hline & & & n-hexane & $2(D)$ & 6.8 & \\
\hline & & & Aqueous & $2(D)$ & 6 & \\
\hline & & & chloroform & $2(D)$ & 5.2 & \\
\hline & & & Butanol & $2(D)$ & 5.1 & \\
\hline C. sativa & $\begin{array}{l}\text { Cherat, Mardan, } \\
\text { Malakand, Kohat }\end{array}$ & \multirow[t]{4}{*}{ Leaves } & Ethanolic & $10(C)$ & 20 & {$[30]$} \\
\hline \multirow[t]{3}{*}{ C. copticum } & \multirow[t]{3}{*}{ Kohat } & & Methanol & $50(\mathrm{D})$ & 10 & \multirow[t]{3}{*}{ [9] } \\
\hline & & & Ethanol & $50(D)$ & 11 & \\
\hline & & & n-hexane & $50(\mathrm{D})$ & 8 & \\
\hline \multirow[t]{3}{*}{ C. Intybus } & \multirow[t]{3}{*}{ Sawabi, Gawadar } & & n-hexane & 20 (D) & 6.3 & \multirow[t]{3}{*}{ [79] } \\
\hline & & & Chloroform & $20(D)$ & 7 & \\
\hline & & & Ethyl acetate & $20(D)$ & 6.3 & \\
\hline \multirow[t]{4}{*}{ C. Intybus } & \multirow[t]{4}{*}{ Mardan } & \multirow[t]{4}{*}{ Roots } & Methanol & $20(D)$ & 7.4 & \multirow[t]{4}{*}[80]{} \\
\hline & & & n-hexane & $20(D)$ & 5 & \\
\hline & & & chloroform & 20 (D) & 6.2 & \\
\hline & & & Ethyl acetate & $20(D)$ & 7 & \\
\hline \multirow{5}{*}{$\begin{array}{l}\text { Cichorium Noenum L. } \\
\text { Asteraceae }\end{array}$} & \multirow[t]{5}{*}{ Sawabi, Gawadar } & & Methanol & $20(D)$ & 3.4 & \multirow[t]{5}{*}{ [79] } \\
\hline & & & n-hexane & 20 (D) & 7 & \\
\hline & & & Chloroform & 20 (D) & 5 & \\
\hline & & & Ethyl acetate & 20 (D) & 5 & \\
\hline & & & Aqueous & 20 (D) & 3.4 & \\
\hline C. zeylanicum & Kohat & & n-hexane & $50(\mathrm{D})$ & 10 & [9] \\
\hline
\end{tabular}


Table 2 Antibacterial activities of Pakistani medicinal plants against $E$. coli at different concentration (Continued)

\begin{tabular}{|c|c|c|c|c|c|c|}
\hline \multirow[t]{15}{*}{ C. tubulosa } & \multirow[t]{15}{*}{ KDA Karak } & & \multirow[t]{2}{*}{ Methanolic } & 4 (D) & 6 & [81] \\
\hline & & & & 2 (D) & 4.1 & \\
\hline & & & \multirow[t]{3}{*}{ Aqueous } & 4 (D) & 4 & \\
\hline & & & & 2 (D) & 3 & \\
\hline & & & & $1(\mathrm{D})$ & 1 & \\
\hline & & & \multirow[t]{2}{*}{ Ethyl acetate } & 4 (D) & 5.5 & \\
\hline & & & & 2 (D) & 3.1 & \\
\hline & & & \multirow[t]{2}{*}{ Chloroform } & 4 (D) & 4.5 & \\
\hline & & & & 2 (D) & 4 & \\
\hline & & & \multirow[t]{3}{*}{ n-hexane } & 4 (D) & 5 & \\
\hline & & & & 2 (D) & 4.1 & \\
\hline & & & & 1 (D) & 3.2 & \\
\hline & & & \multirow[t]{3}{*}{ n-botanol } & 4 (D) & 7 & \\
\hline & & & & $2(D)$ & 6.5 & \\
\hline & & & & $1(\mathrm{D})$ & 5 & \\
\hline \multirow[t]{13}{*}{ C. arvensis } & \multirow[t]{13}{*}{ Peshawar } & \multirow[t]{4}{*}{ Leaves } & Methanol & $0.1(\mathrm{C})$ & 5 & [82] \\
\hline & & & n-hexane & $0.1(C)$ & 6.2 & \\
\hline & & & Chloroform & $0.1(C)$ & 7.1 & \\
\hline & & & Ethyl acetate & $0.1(C)$ & 6.2 & \\
\hline & & \multirow[t]{5}{*}{ Stem } & Methanol & $0.1(C)$ & 4 & \\
\hline & & & n-hexane & $0.1(C)$ & 6 & \\
\hline & & & Chloroform & $0.1(\mathrm{C})$ & 6.2 & \\
\hline & & & Ethyl acetate & $0.1(C)$ & 5.5 & \\
\hline & & & Aqueous & $0.1(\mathrm{C})$ & 3 & \\
\hline & & \multirow[t]{4}{*}{ Root } & Methanol & $0.1(C)$ & 3.2 & \\
\hline & & & n-hexane & $0.1(C)$ & 3.7 & \\
\hline & & & Chloroform & $0.1(C)$ & 5.3 & \\
\hline & & & Ethyl acetate & $0.1(C)$ & 3.3 & \\
\hline \multirow[t]{6}{*}{ D. innoxia } & \multirow{6}{*}{$\begin{array}{l}\text { Mirpur (Azad Jammu } \\
\text { Kashmir) }\end{array}$} & & \multirow[t]{6}{*}{ Methanol } & $3(C)$ & 2 & {$[8]$} \\
\hline & & & & $5(C)$ & 3.5 & \\
\hline & & & & $7.5(C)$ & 4.5 & \\
\hline & & & & $10(C)$ & 6 & \\
\hline & & & & $12.5(C)$ & 7 & \\
\hline & & & & $15(C)$ & 8 & \\
\hline D. regia & Karachi & Flower & Ethanol & $100(C)$ & 10 & [12] \\
\hline D. viscosa & Kohat & Aerial parts & Ethanolic & $3.2(\mathrm{D})$ & 11 & [11] \\
\hline \multirow[t]{5}{*}{ E. gerardiana } & \multirow[t]{5}{*}{ Baluchistan } & \multirow[t]{5}{*}{ Whole plant } & \multirow[t]{5}{*}{ Methanol } & 15 (D) & 2.6 & [77] \\
\hline & & & & $12.5(\mathrm{D})$ & 1.6 & \\
\hline & & & & $10(D)$ & 1.5 & \\
\hline & & & & 7.5 (D) & 1.5 & \\
\hline & & & & 5 (D) & 1.4 & \\
\hline E. camaldulensis & $\begin{array}{l}\text { Cherat, Mardan, } \\
\text { Malakand, Kohat }\end{array}$ & Leaves & Ethanolic & $10(C)$ & 18 & [30] \\
\hline F. carica & $\begin{array}{l}\text { Cherat, Mardan, } \\
\text { Malakand, Kohat }\end{array}$ & Leaves & Ethanolic & $10(\mathrm{C})$ & 23 & [30] \\
\hline
\end{tabular}


Table 2 Antibacterial activities of Pakistani medicinal plants against $\boldsymbol{E}$. coli at different concentration (Continued)

\begin{tabular}{|c|c|c|c|c|c|c|}
\hline \multirow[t]{5}{*}{ G. glabra } & \multirow[t]{5}{*}{ Peshawar } & \multirow[t]{5}{*}{ Root } & \multirow[t]{5}{*}{ Methanol } & $15(D)$ & 3.6 & \multirow[t]{5}{*}{ [77] } \\
\hline & & & & $12.5(\mathrm{D})$ & 1.6 & \\
\hline & & & & $10(\mathrm{D})$ & 1.5 & \\
\hline & & & & 7.5 (D) & 1.4 & \\
\hline & & & & $5(\mathrm{D})$ & 1.4 & \\
\hline \multirow[t]{2}{*}{ H. officinalis } & \multirow{2}{*}{$\begin{array}{l}\text { Azad Jammu } \\
\text { Kashmir }\end{array}$} & \multirow[t]{2}{*}{ Leaves } & \multirow[t]{2}{*}{ Methanol } & $15(\mathrm{D})$ & 1.4 & \multirow[t]{2}{*}{ [77] } \\
\hline & & & & $12.5(\mathrm{D})$ & 1.2 & \\
\hline \multirow[t]{5}{*}{ J. adhatoda } & \multirow[t]{5}{*}{ Margalla Hills } & \multirow[t]{5}{*}{ Leaves } & \multirow[t]{5}{*}{ Methanol } & $15(\mathrm{D})$ & 2 & \multirow[t]{5}{*}{ [77] } \\
\hline & & & & $12.5(\mathrm{D})$ & 1.6 & \\
\hline & & & & $10(\mathrm{D})$ & 1.5 & \\
\hline & & & & $7.5(\mathrm{D})$ & 1.4 & \\
\hline & & & & $5(D)$ & 1.4 & \\
\hline \multirow[t]{4}{*}{ M. neglecta } & \multirow[t]{4}{*}{ Swat } & & Methanolic & $4(D)$ & 13 & \multirow[t]{4}{*}{ [83] } \\
\hline & & & n-hexane & $4(D)$ & 13.5 & \\
\hline & & & Chloroform & $4(\mathrm{D})$ & 14 & \\
\hline & & & Aqueous & $4(\mathrm{D})$ & 10 & \\
\hline \multirow[t]{5}{*}{ M. sylvestris } & \multirow[t]{5}{*}{ Rawal Dam } & \multirow[t]{5}{*}{ Leaves, root, flower } & \multirow[t]{5}{*}{ Methanol } & 15 (D) & 1.8 & \multirow[t]{5}{*}{ [77] } \\
\hline & & & & $12.5(\mathrm{D})$ & 1.6 & \\
\hline & & & & $10(\mathrm{D})$ & 1.6 & \\
\hline & & & & 7.5 (D) & 1.6 & \\
\hline & & & & $5(\mathrm{D})$ & 1.4 & \\
\hline M. longifolia & $\begin{array}{l}\text { Cherat, Mardan, } \\
\text { Malakand, Kohat }\end{array}$ & Leaves & Ethanolic & $10(C)$ & 18 & [30] \\
\hline \multirow[t]{3}{*}{ N. microphyllum. } & \multirow[t]{3}{*}{ Swat } & & n-hexane & 4 (D) & 13 & [83] \\
\hline & & & Chloroform & $4(\mathrm{D})$ & 12 & \\
\hline & & & Aqueous & $4(\mathrm{D})$ & 10 & \\
\hline O. europaea & $\begin{array}{l}\text { Cherat, Mardan, } \\
\text { Malakand, Kohat }\end{array}$ & Leaves & Ethanolic & $10(C)$ & 18 & [30] \\
\hline O. limbata & $\begin{array}{l}\text { Cherat, Mardan, } \\
\text { Malakand, Kohat }\end{array}$ & Leaves & Ethanolic & $10(C)$ & 13 & [30] \\
\hline Otostegia limbata & Abottabad & Aerial parts & Ethanolic & $8(C)$ & 11.5 & [1] \\
\hline & & & methanolic & $8(C)$ & 6 & \\
\hline Phyllanths emblica & Kohat & & Methanol & $50(\mathrm{D})$ & 9.66 & [9] \\
\hline R. communis & Karachi & Leaves & Ethanol & $100(C)$ & 12 & [12] \\
\hline S. surrattense & Mirpur (Azad & & Methanol & $3(C)$ & 1 & [8] \\
\hline & Jammu Kashmir) & & & $5(C)$ & 2.5 & \\
\hline & & & & $7.5(\mathrm{C})$ & 3 & \\
\hline & & & & $10(C)$ & 3.8 & \\
\hline & & & & $12.5(\mathrm{C})$ & 4 & \\
\hline & & & & $15(C)$ & 5 & \\
\hline S. xanthocarpum & Lahore & Leaves, stem, & Ethanolic & $5(C)$ & 11.84 & [84] \\
\hline & & flower, root & & $50(C)$ & 14.52 & \\
\hline & & & & $100(C)$ & 16.78 & \\
\hline T. foenum & Karachi & Seeds & Ethanol & $100(C)$ & 10 & [12] \\
\hline V. album & Azad Jammu Kashmir & Leaves, twigs & Ethyle acetate & $100(C)$ & 24.96 & [85] \\
\hline
\end{tabular}


Table 2 Antibacterial activities of Pakistani medicinal plants against $E$. coli at different concentration (Continued)

\begin{tabular}{|c|c|c|c|c|c|c|}
\hline & & & Chloroform & $100(C)$ & 19.76 & \\
\hline & & & Ethanolic & $100(C)$ & 16.66 & \\
\hline & & & Methanolic & $100(C)$ & 16.93 & \\
\hline & & & Aqueous & $100(C)$ & 9.16 & \\
\hline \multirow[t]{6}{*}{ W. somnifera } & \multirow{6}{*}{\multicolumn{2}{|c|}{$\begin{array}{l}\text { Mirpur (Azad Jammu } \\
\text { Kashmir) }\end{array}$}} & Methanol & $3(C)$ & 1 & [8] \\
\hline & & & & $5(C)$ & 3.7 & \\
\hline & & & & $7.5(C)$ & 4 & \\
\hline & & & & $10(C)$ & 7 & \\
\hline & & & & $12.5(\mathrm{C})$ & 8 & \\
\hline & & & & $15(C)$ & 8 & \\
\hline W. somnifera & $\begin{array}{l}\text { Cherat, Mardan, } \\
\text { Malakand, Kohat }\end{array}$ & Leaves & Ethanolic & $10(C)$ & 18 & [30] \\
\hline Z. vulgaris & Mianwali & Fruits & Methanol & $15(\mathrm{D})$ & 1.4 & [77] \\
\hline
\end{tabular}

$\mathrm{C}=$ Concentration in the respective solvent; $\mathrm{D}=$ Concentration in DMSO solvent.

steroids, glycosides, flavonoids, L-Azetidine-2-carboxylic acid, lupeol etc. have been isolated from the plant when extracted with alcoholic solvents (Table 1).

\section{Dodonaea viscosa}

Traditional healers of Pakistan use mostly leaves' infusion of $D$. viscose for curing different diseases caused by microbial agents (Table 1). The ethanolic extract of aerial parts of $D$. viscose dissolved in DMSO solvent at $3.2 \mathrm{mg} / \mathrm{ml}$ concentration showed $11 \mathrm{~mm}$ inhibition zone [11] against E. coli (Table 2) that might be associated with the presence of tannins, saponins, flavonoids and terpenoids in the studied parts [51].

\section{Medicinal plants extracted with butanol}

Butanolic extracts of Pakistani medicinal plants also showed optimum anti-E. coli inhibitory activities. However, the use of butanol for plant extraction is very limited in Pakistan. Present review reported only 2 plants out of 34 extracted with butanol (Figure 1). Increase in concentration of butanolic extract in DMSO has significantly increased the inhibition zone against $E$. coli (Figure 3).

\section{Calotropis procera}

Traditional healers in the remote areas of Pakistan use $C$. procera in the form of infusion against gastro-intestinal troubles. The butanolic extract of stem and leaves of $C$. procera showed $6 \mathrm{~mm}$ inhibition against $E$. coli at $4 \mathrm{mg} /$ $\mathrm{ml}$ concentration dissolved in DMSO (Table 2). Saponins, alkaloids, triterpenoids and flavonoids classes of compounds might be responsible for its strong anti-E. coli activity [39].

\section{Cistanche tubulosa}

No traditional uses of this plant has been reported in Pakistan, however, in-vitro scientific validation against $E$. coli provide a strong base for this plant to be used as potent medicinal plant. Butanolic extract of C. tubulosa dissolved in DMSO showed 7 and $6.5 \mathrm{~mm}$ inhibition against E. coli at concentration of 4 and $2 \mathrm{mg} / \mathrm{ml}$, respectively (Table 2). Secondary metabolites such as glycosides and monoterpenes have been isolated from the butanolic extracts of this plant.

\section{Medicinal plants' aqueous extracts}

Water is also used as a solvent for the extraction of medicinal plants in Pakistan. Extraction of plants with organic solvents gives more consistent antimicrobial activities as compared to inorganic solvents [89]. Reason behind less activities of inorganic solvent might be due to the presence of better medium for growth and occurrence of microorganisms [90]. Furthermore, water-soluble compounds, such as polysaccharides and polypeptides have no real impact as antimicrobial agents [16]. This could be the main reason of limited use of water for the extraction of medicinal plants in Pakistan. Present review showed that only 2 plants that have been extracted using water as solvent (Figure 1). Negative correlation was found between concentration of aqueous extract of medicinal plants and the zone of anti-E. coli inhibition (Figure 3).

\section{Calotropis procera}

C. procera has shown antibacterial activities due to the presence of different phytoconstituents like flavonoids, tannins etc. Aqueous extracts of the leaves of $C$. procera showed $7.9 \mathrm{~mm}(4 \mathrm{mg} / \mathrm{ml})$ and $6 \mathrm{~mm}(2 \mathrm{mg} / \mathrm{ml})$ inhibition against $E$. coli, when dissolved in DMSO [17]. 


\section{Malva neglecta}

Traditionally the plant is used against gastro-intestinal problems [40]. Aqueous extract of $M$. neglacta showed anti-E. coli inhibition zone of $10 \mathrm{~mm}$ on dissolving in DMSO at $4 \mathrm{mg} / \mathrm{ml}$ concentration [84] (Table 2). No study has been reported on the phytochemical screening of $M$. neglacta (Table 1).

\section{Medicinal plants extracted with ethyl acetate}

Literature review has shown limited use of ethyl acetate for the extraction of medicinal plant in Pakistan against E. coli. Out of 34 medicinal plants, only 2 were extracted with ethyl acetate that showed inhibition against $E$. coli (Figure 1). Significant positive correlation was observed between the concentration of pure ethyl acetate extract and anti-E. coli inhibitory potential (Figure 2). Increase concentration of ethyl acetate in DMSO also increases inhibition potential again $E$. coli, however statistically it not significant (Figure 3).

\section{Viscum album}

Traditionally, local people use $V$. album for variety of ailments like gastro-intestinal and anti-inflammatory (Table 1). The ethyl acetate extract of $V$. album showed $24.96 \mathrm{~mm}$ inhibition against $E$. coli at concentration of $100 \mathrm{mg} / \mathrm{ml}$ that might be due to the presence of variety of active phytoconstituents like 4'-O-[ $\beta$-D-Apiosyl $(1 \rightarrow 2)]$ - $\beta$-D-glucosyl]-5-hydroxyl-7-O-sinapylflavanone, 3(4-acetoxy-3,5-dimethoxy)-phenyl-2E-propenyl- $\beta$-D glucopyranoside and 5,7-dimethoxy-4'-hydroxy flavanone etc. $[91,92]$.

\section{Cichorium intybus}

C. intybus is traditionally used for the treatment of abdominal pain and diarrhea (Table 1). Root extract of C. intybus showed $7 \mathrm{~mm}$ inhibition against $E$. coli at $20 \mathrm{mg} / \mathrm{ml}$ concentration dissolved in DMSO solvent [80]. Antibacterial activity of $C$. intybus might be associated with the presence of different phytoconstituents (Table 2). There is no study reported on the ethyl acetate soluble phytoconstituents in the world. However, methanol soluble phytochemical are [lup-12,20 (29)-dien$3 \beta$-ol-3 $\beta$-L-arabinofuranosyl-2' -hexadecanoate],[lup-12,20 (29)-dien-3 $\beta$-olyl hexadecanoate] and [4 $\beta$-(pent-2-enylolactone)-hexatriacontane] (Table 1).

\section{Medicinal plants extracted with chloroform}

Literature study has indicated chloroform with high inhibition against $E$. coli. In total, 7 medicinal plants were extracted with chloroform (Figure 1). Significant positive correlation was found between anti-E. coli inhibition zone and concentration of plant extracts in their respective solvent.

\section{Malva neglecta}

Ethnomedicinal properties of $M$. neglacta have already been discussed above. Chloroform extract of $M$. neglacta showed $14 \mathrm{~mm}$ zone of inhibition against $E$. coli at $4 \mathrm{mg} / \mathrm{ml}$ when dissolved in DMSO [83] (Table 2).

\section{Cichorium intybus}

Chloroform extract of C. intybus has shown in-vitro inhibitory activity against $E$. coli. It showed $6.22 \mathrm{~mm}$ anti-E. coli inhibition zone at concentration of $20 \mathrm{mg} / \mathrm{ml}$ dissolved in DMSO [80].

\section{Medicinal plants extracted with $\mathrm{n}$-hexane}

$\mathrm{N}$-hexane extracts does not exhibit better anti-E. coli activities. N-hexane extracts of different plants like Terminalia catappa and Dodonaea viscose have been found with no antibacterial activities [11,93]. However, the present review showed that certain plants exhibit antibacterial activities when extracted with n-hexane solvent. In total, 10 out of 34 medicinal plants were extracted with n-hexane showing antibacterial activity (Figure 1), which could be due to difference in the phytochemistry between plants [27].

\section{Cinnamomum zeylanicum}

Traditional importance of C. zeylanicum could be observed due to its varied utilization against different ailments (Table 1). N-hexane extract of C. zeylanicum at $50 \mathrm{mg} / \mathrm{ml}$ in DMSO has shown $10 \mathrm{~mm}$ inhibition against E. coli [9] (Table 2) that might be due to the presence of active phytoconstituents isolated from the bark of C. zeylanicum [45].

\section{Carum copticum}

Ethnomedicinally, C. copticum induces appetite, remove kidney stone as well use for the treatment of digestion and whooping cough. Its seeds are taken with salt for gastric trouble (Table 1). According to Shinwari et al. [9] the n-hexane extract of this plant showed $8 \mathrm{~mm}$ zone of inhibition against the E. coli at $50 \mathrm{mg} / \mathrm{ml}$ in DMSO (Table 2). There is no study conducted so far on the phytochemistry of this plant.

\section{Conclusions}

The present review concluded that inhabitants of remote areas of Pakistan are greatly dependent on ethnomedicinal plants for the treatment of different ailments caused by $E$. coli. Majority of medicinal plants have been proved in-vitro for their therapeutic activities against E. coli. Different organic and inorganic solvents have been used in Pakistan for medicinal plants extraction, however, methanol being used the most. Different compounds such as nimbin, alkaloids of $J$. adathoda; glycosides of W. somnifera etc. were found inhibiting the growth of E. coli. Ethnomedicinal knowledge provides baseline information for 
the search of novel drugs and compounds against variety of infectious diseases cause by microorganisms. Therefore, detailed ethnomedicinal studies should be carried out in Pakistan in order to conserve this valuable knowledge before its extinction. Moreover, solvents other than methanol should also give preference in future as it could lead to the separation of some new therapeutic compounds that could be active against $E$. coli. Phytochemical screening of unexplored plants like $M$. neglecta, C. copticum etc. should be given focussed as it could result in development of new antimicrobial drugs with fewer side effects.

\section{Competing interests}

The authors declare that they have no competing interests.

\section{Authors' contributions}

All authors have fully contributed in writing and revising the manuscript critically. All authors read and approved the final manuscript.

\section{Acknowledgements}

The authors are highly indebted to departmental colleagues for their support.

\section{Author details}

'Department of Botany, Kohat University of Science and Technology, Kohat 26000, Pakistan. ${ }^{2}$ Department of Biotechnology, Quaid-i-Azam University Islamabad, 44000 Islamabad, Pakistan.

Received: 21 March 2014 Accepted: 27 July 2014

Published: 19 August 2014

\section{References}

1. Anwar F, Ali M, Hussain Al, Shahid M: Antioxidant and antimicrobial activities of essential oil and extracts of fennel (Foeniculum vulgare Mill.) seeds from Pakistan. Flavour Fragr J 2009, 24:170-176.

2. Stuffness $\mathrm{M}$, Douros J: Current status of the $\mathrm{NCl}$ plant and animal product program. Nat Prod 1982, 45(1):1-14.

3. Bashir A, Ali N, Bashir S, Choudhary MI: Biological activities of aerial parts of Tylophora hirsuta Wall. Afr J Biotechnol 2009, 8(18):4627-4631.

4. Olayiowola A: WHO's traditional medicine programme: progress and perspective. WHO Chron 1984, 38(2):76-81.

5. Bashir S, Erum A, Kausar R, Saleem U, Ruqia-Tulain U, Alamgeer: Antimicrobial activity of some ethno-medicinal plants used in Pakistan. Res Pharm 2012, 2(1):42-45

6. Hocking GM: Pakistan medicinal plants. Qual Plant Mat Veg 1958, 6:121-136.

7. Ibrar M, Hussain F, Sultan A: Ethnobotanical studies on plant resources of Ranyal Hills, District Shangla. Pakistan Pak J Bot 2007, 2:329-337.

8. Mahmood A, Mahmood A, Tabassum A: Ethnomedicinal survey of plants from District Sialkot, Pakistan. J Appl Pharm 2011, 03:212-220.

9. Shinwari ZK, Salima M, Faisal R, Huda S, Asrar M: Biological screening of Indigenous knowledge based plants used in Diarrheal treatment. Pak J Bot 2013, 45(4):1375-1382.

10. Saroya AS: Herbalism, Phytochemistry and Ethnopharmacology. Enfield, New Hampshire New York: Science Publishers; 2011.

11. Khurram M, Khan MA, Hameed A, Abbas N, Qayum A, Inayat H: Antibacterial activities of dodonaea viscosa using contact bioautography technique. Molecule 2009, 14:1332-1341.

12. Khursheed R, Naz A, Naz E, Sharif H, Rizwani GH: Antibacterial, antimycelial and phytochemical analysis of ricinus communis linn, trigonella foenum grecum linn and delonix regia (Bojer ex Hook.) Raf of Pakistan. Romanian Biotechnol Lett 2012, 17(3):7237-7244.

13. Hussain S, Jamil M, Ullah F, Khan A, Ullah F, Arfan M, Ahmad S, Khatoon L: Antimicrobial and antioxidant activities of the plant Heliotropium strigosum. Afr J Biotechnol 2010, 9(45):7738-7743.

14. Welch RA, Burland V, Plunkett G, Redford P, Roesch P, Rasko D, Buckles EL, Liou SR, Boutin A, Hackett J, Stroud D, Mayhew GF, DRose J, Zhou S, Schwartz DC, Perna NT, Mobley HLT, Donnenberg MS, Blattner FR: Extensive mosaic structure revealed by the complete genome sequence of uropathogenic Escherichia coli. Proc Natl Acad Sci U S A 2000, 99(26):17020-17024.

15. Riley L, Remis W, Helgerson RS: Hemorrhagic colitis associated with a rare Escherichiacoli serotype. N Engl J Med 1983, 308:681-685.

16. Cowan MM: Plant products as antimicrobial agents. Clin Microbiol Rev 1999, 12:564-582.

17. Bibi Z: Animicrobial Activity of Calotrpis Procera. A Thesis Submitted for Fulfillment of Degree of Masters in Chemistry. KUST: Kohat; 2013.

18. Cohen ML: Epidemiology of drug resistance: implications for a post antimicrobial era. Science 1992, 257:1050-1055.

19. Amee R, Manges MPH, James R, Johnson MD, Foxman B, Timothy T, Bryan O, Kathleen E, Fullerton MPH, Lee W, Riley MD: Widespread distribution of urinary tract infections caused by a multidrug-resistant escherichia coliclonal group. N Engl J Med 2001, 345(14):1007-1013.

20. Goettsch W, Ven Pelt W, Nagelkerke N, Hendrix MGR, Buiting AGM, Petite PL, Sabbe LM, Griethuysen AJA, Neeling AJD: Increasing resistance to floroquinolones in E. coli from urinary tract infections in the Netherlands. J Antimicrob Chemother 2000, 46:223-228.

21. Kafaru E: Immense Help Formative Workshop. In Essential Pharmacology. 1st edition. Lagos, Nigeria: Publishers; 1994.

22. Alder JD: Daptomycin, a new drug class for the treatment of Gram-positive infections. Drugs Today 2005, 41:81.

23. Walsh FM, Amyes SGB: Microbiology and drug resistance mechanisms of fully resistant pathogens. Curr Opi Micro 2004, 7:439-444.

24. Bibi Y, Nisa S, Chaudhary FM, Zia M: Antibacterial activity of some selected medicinal.plants of Pakistan. BMC Complement Altern Med 2011, 11(52):1-7.

25. Rauf A, Khan A, Rasool S, Shah ZA, Saleem M: In-vitro antifungal activity of three selected Pakistani medicinal plants. Middle-East J Med Plants Res 2012, 1(2):41-43.

26. Irshad S, Butt $M$, Younus $\mathrm{H}$ : In-vitro antibacterial activity of two medicinal plants Neem (Azadirachta indica) and Peppermint. Int Res J Pharma 2011, 01(01):9-14

27. Ncube NS, Afolayan AJ, Okoh Al: Assessment techniques of antimicrobia I properties of natural compounds of plant origin: current methods and future trends. Afri J Biotechnol 2008, 7(12):1797-1806.

28. Aliero AAJ: Antimicrobial activity of Solanum tomentosum. Afri $J$ Biotechnol 2006, 5(4):369-372

29. Mahmood A, Mahmood A, Mahmood M: In vitro biological activities of most common medicinal plants of family solanaceae. World Appl Sci J 2012, 17(8):1026-1032.

30. Subhan F, Islam T, Asif M, Rehman H: Exploration of Medicinal Plants Activity Against Human Pathogenic Bacteria, A thesis submitted for fulfillment of degree of BS (Hons), Microbiology department. KUST: Kohat; 2013.

31. Matsuda H, Murakami T, Kishi A, Yoshikawa M: Structures of Withanosides I, II, III, IV, V, VI, and VII, new withanolide glycosides, from the roots of Indianwithania somnifera dunal. and inhibitory activity for tachyphylaxis to clonidine in isolated Guinea-Pig lleum. Bioorg Med Chem 2001, 9:1499-1507.

32. Rani S, Khan SA, Ali M: Phytochemical investigation of the seeds of Althea officinalis L. Nat Prod Res 2010, 24(14):1358-1364.

33. Gudej J, Bleganowska ML: Chromatographic investigations of flavonoid compounds in the leaves and flowers of some species of the genus althaea. Chromatographia 1990, 30(5):333-336.

34. Husain SZ, Malik RN, Javaid M, Bibi S: Ethonobotanical properties and uses of medicinal plants of Morgah biodiversity park, Rawalpindi. Pak J Bot 2008, 40(5):1897-1911.

35. Murad W, Azizullah A, Adnan M, Tariq A, Khan KU, Waheed S, Ahmad A: Ethnobotanical assessment of plant resources of Banda Daud Shah, District Karak, Pakistan. J Ethnobiol Ethnomed 2013, 9:77.

36. Sultana S, Khan MA, Ahmad M, Zafar M: Indigenous knowledge of folk herbal medicines by the women of district Chakwal. Pakistan Ethnobot Leaflets 2006, 10:243-253.

37. Ley SV, Denholm AA, Wood A: The Chemistry of Azadirachtin. Nat Prod Rep 1993, 10:109-157.

38. Babadjamiay RC, Faure R, Boukef K, Balansard GAnd E, Vidal E: Arvensoside $A$ and B, Triterpenoid Saponins from Calendula Arvensis. Phytochemistry 1987, 26(6):1785-1788.

39. Hassan SW, Bilbis FL, Lada MJ, Umar RA, Dangoggo SM, Saidu Y, Abubaker MK, Faruk UZ: Evaluation of antifungal activity and phytochemical analysis of leaves, roots and stem barks of Calotropis procera. (Asclepiadaceae). Pak J Biol Sci 2006, 9(4):2624-2629. 
40. Haq F, Habib Ahmad H, Alam M: Traditional uses of medicinal plants of Nandiar Khuwarr catchment (District Battagram), Pakistan. J Med Plants Res 2011, 5(1):39-48.

41. Mahmoud A, ElSohly, Slade D: Chemical constituents of marijuana: the complex mixture of natural cannabinoids. Life Sci 2005, 78:539-548.

42. Turner TE, Elsohly MA, Boeren EG: Constituent of Canabis Sativa L. XVII. A review of the natural constituents. J Nat Prod 2009, 43(2):169-234.

43. Ahmad M, Khan MA, Manzoor S, Zafar M, Sultana S: Check list of medicinal flora of Tehsil Isakhel, District Mianwali-Pakistan. Ethnobotanical Leaflets 2006, 10:41-48.

44. Kumari R, Ali M, Aeri V: Two new triterpenoids fromCichorium intybusL. Roots. J Asian Nat Prod Res 2012, 14(1):7-13.

45. Belemkar S, Kumar A, Pata MK: Pharmacological screening of herbal extract of piper nigrum (Maricha) and Cinnamomum zeylanicum (Dalchini) for anticonvulsant activity. Ethnopharmacology 2013, 2(2):1-5

46. Xie H, Morikawa T, Masuda H, Nakamura S, Muraoka O, Yoshikawa M: Monoterpene constituents from Cistance tubulosa-chemical structures of kankanosides A-E and kankanol. PMID: 16651763 [PubMed-indexed for MEDLINE] 2008.

47. Kocor M, Pyrek JS, Atal CK, Bedi KL, Sharma BR: Triterpenes of Datura innoxia Mill. Structure of Daturadiole and Daturaolone. J Organic Chem 1973, 38(21):3685-3688.

48. Roy SP, Gupta R, Bhadra D, Kannadasan T: A review on some indigenous medicinal plants with hepatoprotective activity. J Chem Pharma Sci 2013, 6(2):85-92.

49. Jahan I, Rahman MS, Rahman MZ, Kaisar MA, Islam MS, Wahab A, Rashid MA: Chemical and biological investigations Of Delonix regia (Bojer ex Hook.). Raf Acta Pharm 2010, 60:207-215.

50. Sung ML, Fowden L: Azetidine-2-carboxylic acid from the legume Delonix regia. Phytochemistry 1969, 8(10):2095-2096.

51. Prakash NKU, Selvi CR, Sasikala V, Dhanalakshmi S, Prakash SBU: Phytochemistry and Bio-Efficacy of a weed, Dodonaea viscosa. Int J Phar Pharma Sci 2012, 4(2):509-512.

52. Singab AN, Ayoub N, Eman A-S, Martiskainen O, Sinkkonen J, Pihlaja K: Phenolic constituents of eucalyptus camaldulensis Dehnh, with potential antioxidant and cytotoxic activities. Rec Nat Prod 2011, 5(4):271-280.

53. Kalaskar MG, Shah DR, Raja NM, Surana SJ, Gond NY: Pharmacognostic and Phytochemical Investigation of Ficus carica Linn. Ethnobotanical Leaflets 2010, 14:599-609.

54. Varsha S, Agrawal RC: Glycyrrhiza glabra- a plant for the future. Mintage J Pharma Med Sci 2013, 2(3):15-20.

55. Matsuuraa H, Miyazaki H, Asakawa C, Amano M, Yoshihara T, Mizutani J: Isolation ofa-glusosidase inhibitors from hyssop (Hyssopus officinalis). Phytochemistry 2004, 65:91-97.

56. Wang N, Yang XW: Two new flavonoid glycosides from the whole herbs of Hyssopus officinalis. J Asian Nat Prod Res 2010, 12(12):1044-1050

57. Geone MC, Alcântara AF De C: Chemical constituents and biological activities of species of Justicia- a review. Revista Brasileira De Farmacognosia Brazilian J Pharmacognosy 2011, 22(1):220-238.

58. Kumar A, Ram J, Samarth RM, Kumar M: Modulatory influence of Adhatoda vasica Nees leaf extract against gamma irradiation in Swiss albino mice. Phytomedicine 2005, 12:285-293.

59. Ilahi I, lqbal Z, Rehman SU: Cistanche tubulosa (Schenk) r. wight an important medicinal plant occurring in sand dunes of Karak, N.W.F.P. Pakistan. Pak J Bot 2010, 42(1):537-547.

60. Veshkurova O, Golubenko Z, Pshenichnov E, Arzanova I, Uzbekov V, Sultanova E, Salikhov S, Williams HJ, Reibenspies JH, Puckhaber LS, Stipanovic RD: Malvone A, a phytoalexin found inMalva sylvestris (family Malvaceae). Phytochemistry 2006, 67:2376-2379.

61. Ali MS, Saleem M, Ahmad W, Parvez M, Yamdagni R: A chlorinated monoterpene ketone, acylatedb-sitosterol glycosides and a flavanone glycoside from Mentha longifolia (Lamiaceae). Phytochemistry 2002, 59:889-895.

62. Sharaf M, Ansari MAE, Saleh NAM: Flavone glycosides fromMentha longifolia. Fitoterapia 1999, 70:478-483.

63. Khan MY, Panchal S, Vyas N, Butani A, Kumar V: Olea europaea: a phyto-pharmacological review. Pharmacog Rev 2007, 1(1):112-116.

64. Benavente GJ, Castillo J, Lorente A, Ortuno A, Del Rio JA: Antioxidant activity of phenolics extracted from Olea europaea L. leaves. Food Chem 2000, 68:457-462.
65. Khan A, Ahmad VU, Farooq U, Bader S, Arshad S: Two new flavonol glycosides from otostegia limbata benth. Chem Pharm Bull 2009, 57(3):276-279.

66. Shao-Hua W, Wub DG, Chen YW: Chemical constituents and bioactivities of plants from the genus paeonia. Chem Biodivers 2010, 7:90-104.

67. Jan S, Khan MA, Din SU, Murad W, Hussain M, Ghani A: Herbal Rmidies used for gastro-intestinal disorders in Kaghan Valley, NWFP, Pakistan. Pak J Weed Sci Res 2008, 14(3-4):169-200.

68. Riaz N, Anis I, Malik A, Ahmed Z, Rehman AU, Muhammad P, Nawaz SA, Choudhary MI: Paeonins A and B, lipoxygenase inhibiting monoterpene galactosides from Paeonia emodi. Chem Pharm Bull 2003, 51(3):252-254.

69. Dhale DA, Mogle UP: Phytochemical screening and antibacterial activity of phyllanthus emblica (L.). Sci Res Rep 2011, 1(3):138-142.

70. Rehman HU, Yasin KA, Choudhary MA, Khaliqy N, Rahman AU, Muhammad lqbal Choudhary MI, Malik S: Studies on the chemical constituents of Phyllanthus emblica. Nat Prod Res 2007, 21(9):775-781.

71. Singh PP, Ambika, Chauhan SMS: Activity guided isolation of antioxidants from the leaves of Ricinus communis L. Food Chem 2009, 114:1069-1072.

72. Singh OM, Singh TP: Phytochemistry of Solanum xanthocarpum: an amazing traditional healer. J Sci Ind Res 2010, 69:732-740.

73. Singh OM, Subharani K, Singh NI, Devi NB, Nevidita L: Isolation of steroidal glycosides from Solanum xanthocarpum and studies on their antifungal activities. Nat Prod Res 2007, 21(7):585-590.

74. Guo-Rong Wang GR, Tang WZ, Yao QQ, Zhong H, Liu YJ: New flavonoids with $2 \mathrm{BS}$ cell proliferation promoting effect from the seeds ofTrigonella foenum-graecum L. J Nat Med 2010, 64:358-361.

75. Abbasi AM, Khan SM, Ahmad M, Khan MA, Quave CL, Pieroni A: Botanical ethnoveterinary therapies in three districts of the Lesser Himalayas of Pakistan. J Ethnobiol Ethnomed 2013, 9(1):84. 1-20.

76. Biswas K, Chattopadhyay I, Banerjee RK, Bandyopadhyay U: Biological activities and medicinal properties of neem (Azadirachta indica). Curr Sci 2002, 82(11):1336-1345.

77. Walter C, Shinwari ZK, Afzal I, Malik R: Antibacterial activity in herbal products used in pakistan. Pak J Bot 2011, 43:155-162

78. Aslam F, Rehman KU, Asghar M, Sarwar M: Antibacterial activity of various phytoconstituents of Neem. Pak J Agri Sci 2009, 46(3):209-213.

79. Ahmad A: Antimicrobial, Antioxidative and Photochemical Evaluation of Cichorium Intybus and Related Species, A thesis submitted for fulfillment of degree of MASTERS of Philosophy in Chemistry. KUST: Kohat; 2010.

80. Sattar Fl: Antibacterial and Antifungal Activities of Cichorium Intybus, A thesis submitted for fulfillment of degree of Masters in Chemistry. Kohat: kust; 2010.

81. Rehman A: Antibacterial and Anti-Fungal Activity of Medicinal Plant Cistanche Tubulosa, A thesis submitted for fulfillment of degree of M.sc, chemistry department. KUST: Kohat; 2013.

82. Raza M, Fozia, Rehman A, Wahab A, lqbal H, Ullah H, Ahmad S, Ahmad I, Shah SM: Comparative antibacterial study of convolvulus arvensis collected from different areas of Khyber Pakhtunkhwa, Pakistan. Int Res J Pharm 2012, 3(10):220-222

83. Imtiaz B: Antimicrobial Activity of Malva Neglecta and Nasturtium Microphyllum, A thesis submitted for fulfillment of degree of BS (Hons), chemistry department. KUST: Kohat; 2011.

84. Ghani MU, Farooq MU, Khan MTJ: Phytochemical investigations and evaluation of antibacterial and Irritant potential of different extract of whole plant of solanum xanthocarpum schrd and wendl. J Chin Chem Soci 2010, 57:1257-1262.

85. Hussain MA, Khan MQ, Hussain N: Antimicrobial screening of viscum album L. extracts. 2nd Int Conf Environ Sci Technol IPCBEE 2011, 6:203-208,

86. Rachana, Sujata B, Mamta P, Manoj KP, Sonam S: Review and future perspectives of using vasicine, and related compounds. IGJPS 2011, 1:85-98.

87. Papachristos DP, Stamopoulos DC: Repellent, toxic and reproduction inhibitory effects of essential oil vapours on Acanthoscelides obtectus (Say) (Coleoptera: Bruchidae). J Stored Prod Res 2002, 38:117-128.

88. Samarth RM, Kumar A: Mentha piperita (Linn) leaf extract provides protection against radiation induced chromosomal damage in bone marrow of mice. Indian J Exp Biol 2003, 41:229-237.

89. Tiwari P: Phytochemical screening and extraction: a review. Int Phar Sci 2011, 1(1):98-106

90. Lapornik B, Prosek M, Wondra AG: Comparison of extracts prepared from plant by-products using different solvents and extraction time. J Food Eng 2005, 71:214-222. 
91. Shelar P, Reddy SVK, Shelar SGS, Reddy GVS: Medicinal value of mangroves and its antimicrobial properties - a review. Continental J Fisheries Aquatic Sci 2012, 6(1):26-37.

92. Choudhary MI, Maher S, Begum A, Khan AA, Ali S, Khan A, Rehman SU, Rahman AU: Characterization and antiglycation activity of phenolic constituents from Viscum album (European Mistletoe). Chem Pharm Bull 2010, 58(7):980-982.

93. Muhammad, Mudi SY: Phytochemical screening and antimicrobial activities of Terminalia catappa. Leaf Extracts 2011, 23(1):35-39.

doi:10.1186/s12941-014-0040-6

Cite this article as: Adnan et al.: Ethnomedicinal and phytochemical

review of Pakistani medicinal plants used as antibacterial agents against Escherichia coli. Annals of Clinical Microbiology and Antimicrobials

2014 13:40

\section{Submit your next manuscript to BioMed Central and take full advantage of:}

- Convenient online submission

- Thorough peer review

- No space constraints or color figure charges

- Immediate publication on acceptance

- Inclusion in PubMed, CAS, Scopus and Google Scholar

- Research which is freely available for redistribution 